\title{
Data-driven model for maintenance decision support - a case study of railway signalling systems
}

\author{
Amparo Morant ${ }^{1}$, Per-Olof Larsson-Kråik ${ }^{1,2}$, Uday Kumar ${ }^{1}$ \\ 1 Luleå Railway Research Center, Operation and Maintenance Engineering, Luleå University of \\ Technology, SE-971 87, Luleå, Sweden \\ ${ }^{2}$ Trafikverket (the Swedish Transport Administration), Sweden
}

Corresponding author: amparo.morant@1tu.se,+46722446769

\begin{abstract}
Signalling systems ensure the safe operation of the railway network. Their reliability and maintainability directly affect the capacity and availability of the railway network, in terms of both infrastructure and trains, as a line cannot be fully operative until a failure has been repaired. The purpose of this paper is to propose a data-driven decision support model which integrates the various parameters of corrective maintenance data and to study maintenance performance by considering different RAMS parameters. This model is based on failure analysis of historical events in the form of corrective maintenance actions. It has been validated in a case study of railway signalling systems and the results are summarised. The model allows the creation of maintenance policies based on failure characteristics, as it integrates the information recorded in the various parameters of the corrective maintenance work orders. The model shows how the different failures affect the dependability of the system: the critical failures indicate the reliability of the system, the corrective actions give information about the maintainability of the components, and the relationship between the corrective maintenance times measures the efficiency of the corrective maintenance actions. All this information can be used to plan new strategies of preventive maintenance and failure diagnostics, reduce the corrective maintenance, and improve the maintenance performance.
\end{abstract}

\section{Keywords}

Railway, signalling systems, decision support model, data-driven model, failure analysis, maintenance, corrective maintenance, dependability, case study, RAMS, data integration

\section{INTRODUCTION}

The railway network is a complex system with several technologies working together to handle the increasing demands on capacity, speed and mobility for the transportation of goods and passengers. Railway infrastructure managers need planning tools that will enable them systematically to analyse and optimise budget needs, minimise the total costs while achieving the required levels of reliability, availability, maintainability and safety (RAMS), and guarantee the quality of the railway assets in the long run [1]. In order to deal with the short-term cost and performance demands and to guarantee RAMS over time, systematic maintenance management of the railway assets is needed [2]. The EN 50126 standard [3] establishes the processes for the specification and demonstration of RAMS requirements in the railway network.

Railway signalling systems are composed of several different sub-systems, each with its own purpose; however, their interoperability is crucial to the signalling system as a whole. Previous studies have shown the importance of signalling systems for the dependability of the railway network $[4,5]$. Since signalling systems ensure the safe operation of the railway network, their reliability and maintainability directly 
affect the capacity and availability of the railway network, in terms of both infrastructure and trains. The functionality of the signalling system is based on the principle of "fail safe" operation, meaning that the railway section where a failure is located will not be fully operative until the failure has been repaired (since safety cannot otherwise be ensured); hence, the dependability of the signalling system directly affects the capacity of the network.

Rail industry records show that for common railway signalling assets, the occurrence of no-fault-found (NFF) events can be as high as 50\% [5]. The high amount of NFF events can be attributed to a limited understanding of the root causes and characteristics of failures on complex systems, inappropriate means of diagnosing the condition of the systems, and the inability to duplicate the field conditions [6]. Some research shows the importance of NFF events, not just technically, but also organisationally and behaviourally, and proposes addressing this issue as an integrated problem [5,7,8,9]. Granström [10] describes how the number of NFF events of the railway signalling systems can be reduced by updating the maintenance requirements for these systems. Another important cause of failure is human error when performing corrective maintenance $[11,12]$. Other external factors such as environmental conditions can affect the number of corrective maintenance actions $[13,14]$. For a holistic picture of where failures are located and the dominant factors causing them, structured databases containing the complete information are required [15]. A failure analysis based on the empirical data recorded on the corrective maintenance work orders (WOs) would take the external factors into account and be able to measure their relative importance for the dependability of the system.

Maintenance managers responsible for deciding maintenance actions face an abundance of data and have a complicated task transforming these data into information that supports maintenance actions [16]. Failure analysis of a signalling system will give information about how this system is affecting the dependability of the operation and maintenance of the railway network, and identify possible areas of improvement. Pecht and Ramappan [17] find that the primary objective of failure analysis is to provide design feedback to improve the performance of the component. A number of proposals have been made to improve maintenance support performance by analysing maintenance data $[18,19]$.

Two approaches can be considered when studying the performance of a system. While the intrinsic RAMS only depends on the design of the asset and the time required to perform a corrective action with no external factors involved, the operational RAMS takes into account the maintenance performance and the external factors particular to the location (the environment, human factors, random failures, logistics, etc.). While for the intrinsic approach a model could be based on the theory of failure, by studying the different components and the theory of failure for each of them, for the operational approach a datadriven model would be a better choice, since the corrective maintenance records reflect the performance of the system with all the external factors involved in the operation and maintenance. Moreover, since signalling systems are a combination of mechanical, electronic and electrical components, the theory of failure becomes very complex to model. A data-driven model can reflect the failure phenomena with greater accuracy than models based on the theory of failure.

The EN 50129 standard [20] lists methods to identify and evaluate the effects of faults on railway signalling systems, including failure mode, effects and criticality analysis (FMECA), fault tree analysis (FTA), and other methods based on historical data, i.e. common cause failure analysis and historical event analysis. We propose a decision support model for maintenance policies based on data-driven failure analysis of the corrective maintenance. A data-driven model will consider all the factors when a system is in operation (e.g. the environment, human error, etc.), making it possible to quantify the probability that failure will occur. It can be performed on a software-based platform using the WOs already recorded as input. 
The purpose of this paper is to propose a data-driven decision support model which integrates the various parameters of corrective maintenance data and to study the maintenance performance by considering different RAMS parameters. This model is based on failure analysis of historical events in the form of corrective maintenance actions. It has been validated in a case study of railway signalling systems and the results are presented. The model allows the creation of maintenance policies based on failure characteristics, as it integrates all the information recorded in the various parameters of the corrective maintenance WOs. All this information can be used to plan new strategies of preventive maintenance, reduce the corrective maintenance, and improve the maintenance and operating performance.

\section{RESEARCH METODOLOGY}

Corrective maintenance records formed the basis of the analysis. A schematic diagram of the research methodology is shown in Figure 1. Internal documentation, a literature review and interviews with experts were key to interpreting the data and discussing the results of the model. The research is based on data obtained from Trafikverket (the Swedish infrastructure manager). Corrective maintenance WOs related to the railway infrastructure in Sweden are managed in Trafikverket's failure reporting system, called "Ofelia". This system is based on the BMC Software Remedy, which was adapted to the specific requirements of the management of corrective maintenance for railway infrastructure. Ofelia has its own manual for data analysis [21].

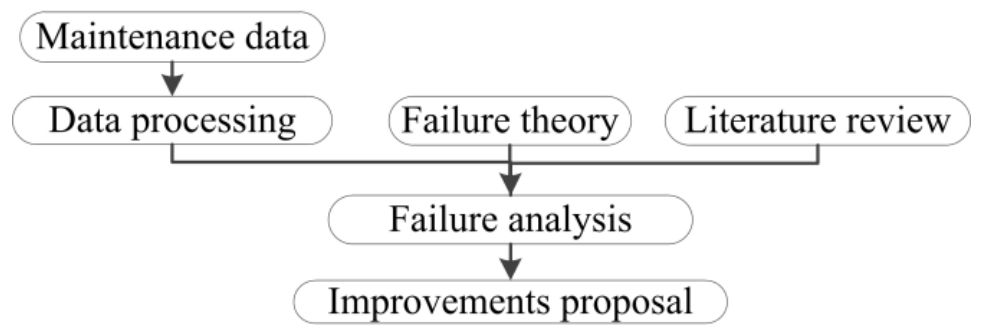

Figure 1: Research methodology process

The process of failure reporting is described in a document published by Trafikverket [22] and specifying the proper procedure from the point in time when a failure is identified and reported to the point in time when the corrective action is finished and the WO related to the failure is closed. A number of partners are involved in the process: the failure can be identified by the train operator, the railway infrastructure manager controls the activity in the railway network, and a subcontracted company performs the corrective maintenance. Since some parameters in 0felia are registered manually and the data can be in several formats, processing the data is necessary to group the information into the correct parameters.

The IEC 61703 standard [23] relates the performance aspects of maintenance to the maintenance variables to measure the dependability of a system. In our case, based on the information recorded in Ofelia, we consider the parameters that give more information about failures. In order to identify these parameters, we performed an exploratory analysis looking at the quality of the data recorded for each parameter (the amount of data recorded for each parameter and the quality of the information). This was needed because not all the parameters are indicated in a WO in 0felia.

Manuals, standards and interviews with experts helped define the parameters for our model [21,22,24]. Parameters with a low data quality (information not recorded or incomplete information) were discarded. The chosen parameters were analysed and the information was integrated to obtain the maximum possible information on corrective maintenance. We studied the values of the parameters themselves, their interrelations with other parameters, and the variations over time. 
Corrective maintenance WOs were gathered from Trafikverket's corrective maintenance database and then processed. The R software was used for the data processing and posterior analysis [25]. The failure modes, causes of failures and corrective actions were related to identify possible improvements, such as establishing an action procedure list for corrective maintenance performance. The times to restore were analysed to find patterns and to compare the total time to maintain (TTM) with the time to restore (TTR).

A case study was used to validate the model; some of the results are summarised in this paper. Based on the analysis, we could identify the weakest points, such as a low reliability of components, problems with information accessibility, a high failure frequency, high times to restore or/and maintain, problems identifying failures, etc. The analysis allowed us to see where the general maintenance performance could be improved.

It is assumed that all the failures were recorded in the corrective maintenance database. One limitation of this research is that the failure data are related to a specific railway corridor, with specific environmental and operational characteristics. Since this research is based on empirical data, the results are limited to the information that could be obtained from the recorded data. Finally, the results showed here are limited to the system asset level for simplification.

\section{MODEL DESCRIPTION}

To develop our model, we considered the different parameters recorded on the corrective maintenance WOs as the inputs for our model. Based on the results of the exploratory analysis of the corrective maintenance records, we included the following parameters in our model:

- Symptom: a symptom that identifies a failure and triggers the opening of a WO, usually observed by the train driver and defined by reference to the system asset affected.

- System asset/subsystem asset: the asset where corrective action is performed.

- Failure mode: the real failure which is related to the corrective action.

- Cause of failure: the reason for the failure.

- Corrective action: the action performed in order to close the WO.

- Failure date: the date when the WO was generated.

Some conclusions can be made based on the times spent on the different WOs. From the database, we can extract the following times and dates for the corrective maintenance WOs: the times and dates for the failure identification, the opening of the WO, the start of the corrective action, the completion of the corrective action, and the closure of the WO. Our objective is not only to study the mean values for each parameter, but also to determine how the values are distributed. This will allow us to analyse how the maintenance performance is affected by external factors. We define three possible states for the assets during operation:

- The available state, corresponding to the up-time (UT): the system is fully operative.

- The unavailable state corresponding to the waiting time (WT): the time between the failure occurrence and the start of the corrective action. During the waiting time, the WO is opened, the failure is identified, the maintenance personnel are informed, the spare parts and tools are gathered, and the personnel go to the location of the failure.

- The unavailable state corresponding to the restoration time (RT): corrective actions are performed and the WO is closed.

Based on these three states, we define the following parameters: the time to maintain (TTM), which is the total downtime (DT) when the system is not available for operation; the time to failure (TTF), which is the time when the system is available for operation without a failure; and the relative restoration time 
(RRT), which is the ratio between the restoration time and the total downtime (see Equations 1, 2 and 3). Figure 2 shows the correspondence between these parameters.

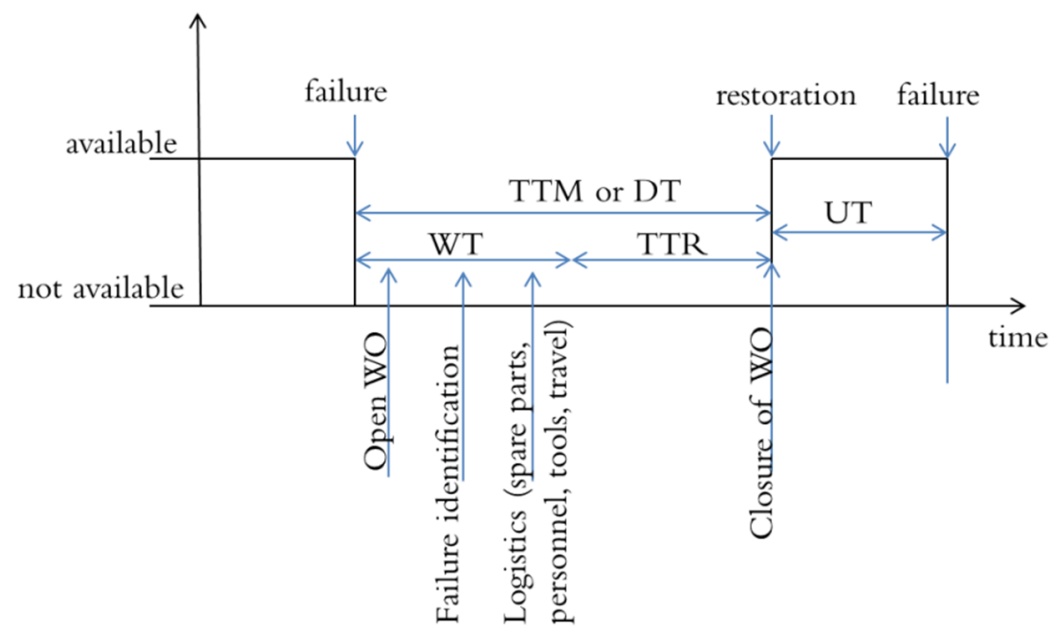

Figure 2: Correspondence between times

$\mathrm{TTM}=\mathrm{t}($ finish of corrective action $)-\mathrm{t}$ (failure identification)

$\mathrm{TTR}=\mathrm{t}($ finishof corrective action $)-\mathrm{t}$ ( startof corrective action)

$\operatorname{RRT}(\%)=\frac{\operatorname{TTR}(\mathrm{sec})}{\operatorname{TTM}(\mathrm{sec})}$

The number of failures in a determined interval will give a measure of the reliability of the system, while the TTR and TTM will indicate the maintenance support performance and the up-time and downtime will show the maintainability [23]. The cause of failure and the failure mode permit us to analyse the common cause failures, and with the failure data we can analyse the historical events. An analysis of the relationship between the various parameters on the corrective maintenance WOs yields insight into the RAMS parameters of the system and allows us to evaluate the maintenance performance of each asset.

Figure 3 shows the proposed decision support model based on the maintenance records. Different outputs are obtained. The most important are the measurement of failure rate, availability and maintainability performance not necessarily related to the system or subsystem asset. This provides a multidimensional tool for measuring the corrective maintenance and the parameters associated not depending on the asset affected. The link of the same corrective actions on different assets allows comparing procedures and identifying best practices.

The relation between causes of failure and failure modes allows identifying the factors most affecting the reliability of the signalling system; further actions can be oriented to reduce those causes. The relationships between the different parameters, such as the relation between failure modes and corrective actions, and the RRT provide insight of the maintenance performance for the different failure modes and assets, which can give indications of which are the areas where an improvement on the would provide the most benefits in terms of maintenance optimization. The relation between failure modes and failure causes can help to identify possible failure modes that could be suitable to consider for condition based maintenance $(\mathrm{CBM})$ in order to reduce the corrective maintenance actions. 


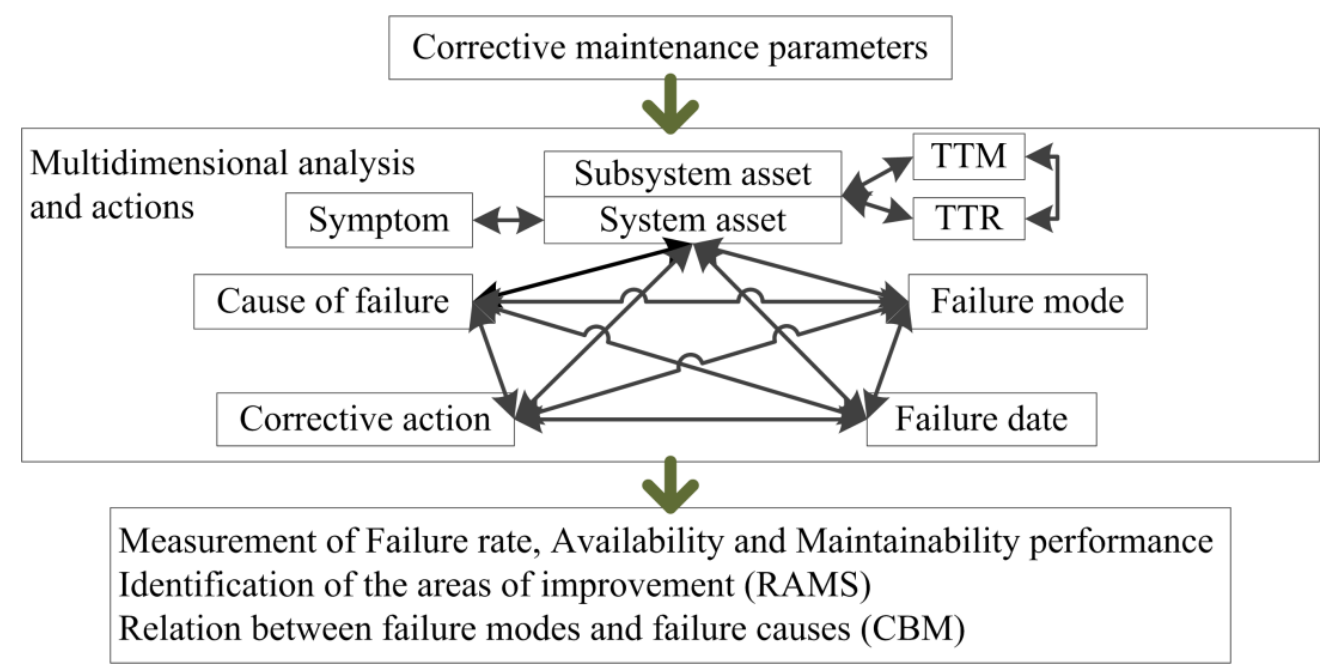

Figure 3: Decision support model based on failure analysis

\section{MODEL IMPLEMENTATION}

The model can facilitate the continuous improvement of maintenance to meet dependability standards [26]. Because Trafikverket considers standards of dependability and RAMS [3, 26] in its maintenance strategies, this model can be implemented as a part of its maintenance assessment process to measure maintenance performance, analyse the results and determine further actions for dependability improvement (see Figure 4).

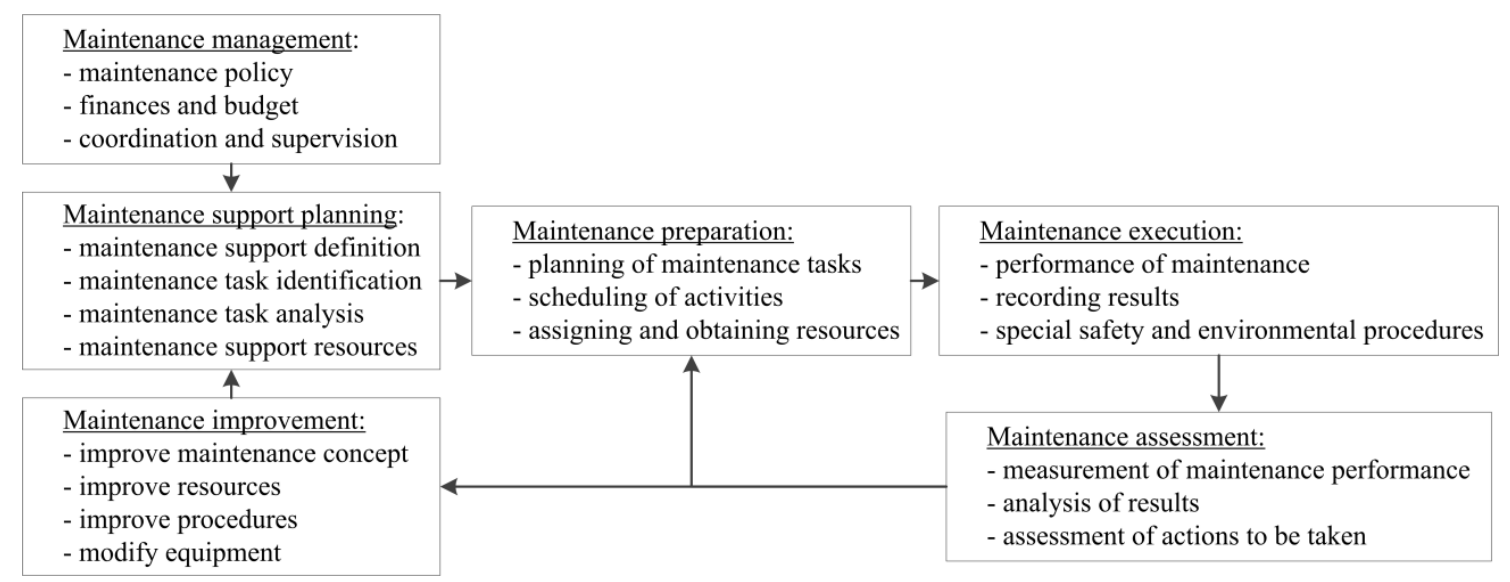

Figure 4: Maintenance processes [26]

This model identifies possible areas for improvement in dependability, allowing companies to review their maintenance planning, preparation and execution. The model considers how various changes in maintenance policies (e.g. preventive inspections, corrective maintenance procedures, failure identification, etc.) affect the dependability of the systems (e.g. increasing availability and reliability, reduce TTR, etc.). 
Finally, the model can also be an input when studying different maintenance policies and their life-cycle cost (LCC) analyses. There are some uncertainties in LCC calculations due to the statistical characteristics of reliability and maintainability parameters, such as the MTTF and MTTR [27]. LCC analyses can be utilised to focus on maintenance strategies to minimise the maintenance cost in the system's life cycle, while meeting dependability requirements. Optimising the maintenance policy is a critical factor in achieving cost effectiveness of the system in the long run. All such optimisations should aim at maximum system availability and minimum LCC and, in the case of Trafikverket, minimum train delays for a specific traffic scenario [28].

Even though this paper focuses on signalling systems in the Swedish railway, the model could be adapted to other railway systems or other networks. In the case of other systems in the Swedish railway, this would be directly applicable, since the information recorded in 0felia is the same for all assets that are part of the larger infrastructure. In the case of other railway networks or systems, the model remains valid, since the parameters considered constitute the basis for understanding and studying the dependability of any system; the only limitation would be the availability of maintenance records required to implement the model.

\section{CASE STUDY}

The analysis is based on a fully operative railway line where the ATC (automatic train control) signalling system supervises and controls the network. The line has been operative with no major changes for many years; hence, we can assume that the WOs represent maintenance and not design changes or failures. The data cover the WOs from January 2003 until November 2012 on a $203 \mathrm{~km}$ long line in the northern part of Sweden.

More specifically, 9,030 WOs were registered during that period, of which 2,455 were associated with signalling systems. Due to the number of WOs directly related to signalling systems $(27 \%)$ and in consideration of the criticality of the good performance of the signalling systems, we focused our research on these systems, even though the methodology can be extrapolated to the whole railway network.

The signalling system in the corridor investigated in this case study is composed of the following systems [24]:

- $\quad$ Track circuits, which are responsible for the train location.

- Balise groups, which give input from the track to the on-board signalling system (e.g. speed limits, driving mode, etc.).

- Level crossings, which coordinate the road traffic crossing the railroad.

- Signals, which give permission to or place restrictions on the trains coming into a track section.

- Signalling boards, which give fixed information to trains (e.g. tunnels, bridges, speed restriction areas, etc.).

- Traffic management system (TMS), which is the interface between the traffic operator and the railway network.

- Interlockings (IXLs) and radio block centre (RBC), which receive the input from the different systems (e.g. track circuits, level crossings, signals and the TMS), perform calculations and return as an output the train operation restrictions to ensure safe operation.

Every WO has associated values for the analysed parameters. The values found for each parameter include values for failure modes, causes of failure and corrective actions. More values can be considered, but since this research is based on empirical data, it focuses on the causes of failure for the signalling systems recorded in the corrective maintenance data. 
The values found for the parameter of failure mode are as follows: "not defined" (the failure mode is not specified in the WO), "non-operative" (the asset is not working properly), "NFF" (no failure was found), "electrical", "mechanical" and "external".

The causes of failure for the signalling systems recorded in the corrective maintenance data include the following:

- Design (e.g. improper design/installation/mounting, etc.);

- Electrical causes, such as electrical overstress, improper isolation, abnormal feeding, power failure, etc.;

- Environmental causes, such as strong winds, extreme temperatures, thunderstorms, snow, ice, etc.;

- External reasons (e.g. railway vehicles, obstacles, third party work, etc.);

- $\quad$ Lack of maintenance or incorrect operation;

- Mechanical reasons, such as fatigue, wear, mechanical overstress, etc.;

- $\quad$ NFF - no failure found (it was impossible to find any failure);

- Not defined (unknown reason), which is recorded when the failure cannot be defined or is not described on the WO.

When the failure of a signalling system asset occurs, the different possible corrective actions performed to return it to the optimal state are classified into the following groups:

- $\quad$ Repair or replacement (restoration) of the failed component;

- $\quad$ Restart/software (SW) update when the failure is attributed to SW causes;

- Provisional repair, but further corrective actions should be scheduled;

- Adjustment/lubrication between modules/connections;

- Cleaning or removal of obstacles (due to an external factor or dust accumulation);

- Control of the system (it is not considered to need repair or replacement, or the failure could not be found, but certain symptoms indicate possible future failure);

- $\quad$ Not defined (the action performed is not specified in the WO);

- No action performed (it is considered that the system does not need the repair or replacement of a component, or the failure could not be found).

When studying the time to restore in the WOs, we found that, of the 2,456 WOs related to failures of signalling systems, 103 WOs had a restoration time of 0 seconds. Only 19 of these had a corrective action which could be used to calculate the restoration time, such as "repair" (1 WO), "replacement" (10 WOs), "restart" (3 WOs), and "removal of obstacles" ( 3 WOs). We decided not to consider these data, as their omission would not greatly affect the results of the analysis. The other abnormal result was that one WO had a negative time, probably due to an error when writing the "correction action start date".

Approximately $16 \%$ of the WOs have large times to restoration and maintain (more than one day). This can be due to different factors; e.g. the failure may not have affected the normal operation of the railway network and could wait for other scheduled maintenance; the complexity of the restoration may have been high; or it may have been difficult to identify where the failure was, etc. The procedures for corrective maintenance at Trafikverket state that a WO should be closed within a maximum of 24 hours [22]. Hence, we discarded the WOs which were open for longer than 24 hours.

A preliminary analysis could be made by determining the values for each of the parameters that were more important (considering the WOs that comprised $80 \%$ of the total). However, to state the maintenance characteristics and needs for each asset, we not only had to examine the total number of failures during the time frame of our sample, but also had to consider the yearly occurrences. This helped to show if the failures had occurred in one particular year (caused by specific environmental factors, for 
example), or if the results could be generalised. To study how the different parameters were related for the different assets in the railway signalling systems, we needed to find trends and differences in behaviour related to where the failure had occurred. The results of our analysis varied and are summarised in the next sections, together with a discussion of how to improve the dependability of the system based on our analysis.

\section{RESULTS}

Figure 5 shows the Pareto diagrams for the chosen parameters (system asset, failure mode, cause of failure and corrective action); these parameters and the relationship between them will be summarised in this section.

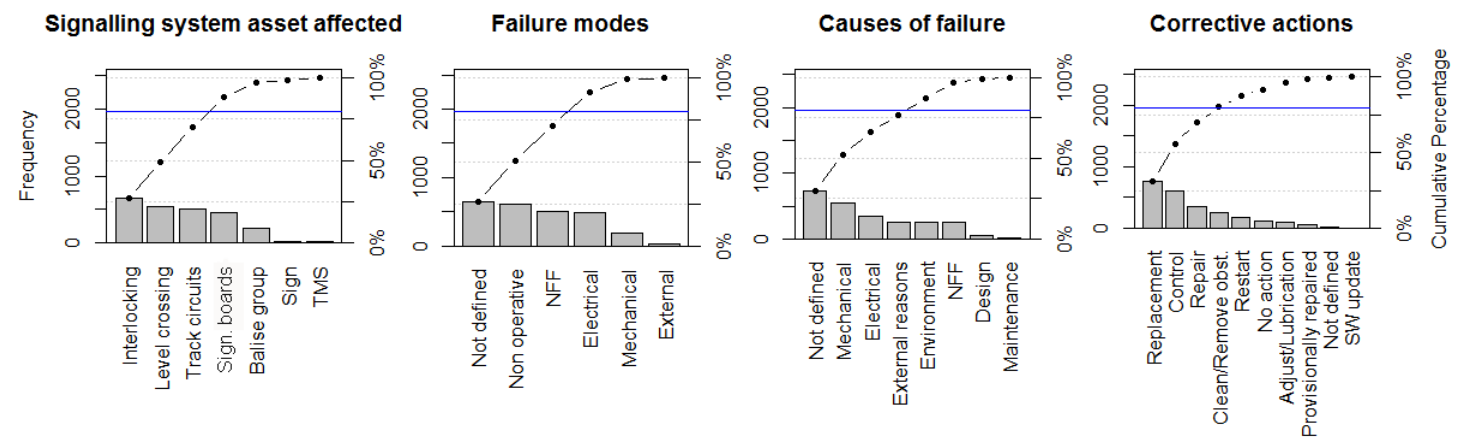

Figure 5: Pareto graphs showing the values for the different parameters: (a) signalling system asset affected; (b) failure modes; (c) failure causes (d) corrective actions performed

\subsection{The parameter of system asset affected}

When examining the different system assets that comprise signalling systems, we found that the interlockings, the level crossings, the track circuits and the signals account for more than $80 \%$ of the failures overall (see Figure 5a).

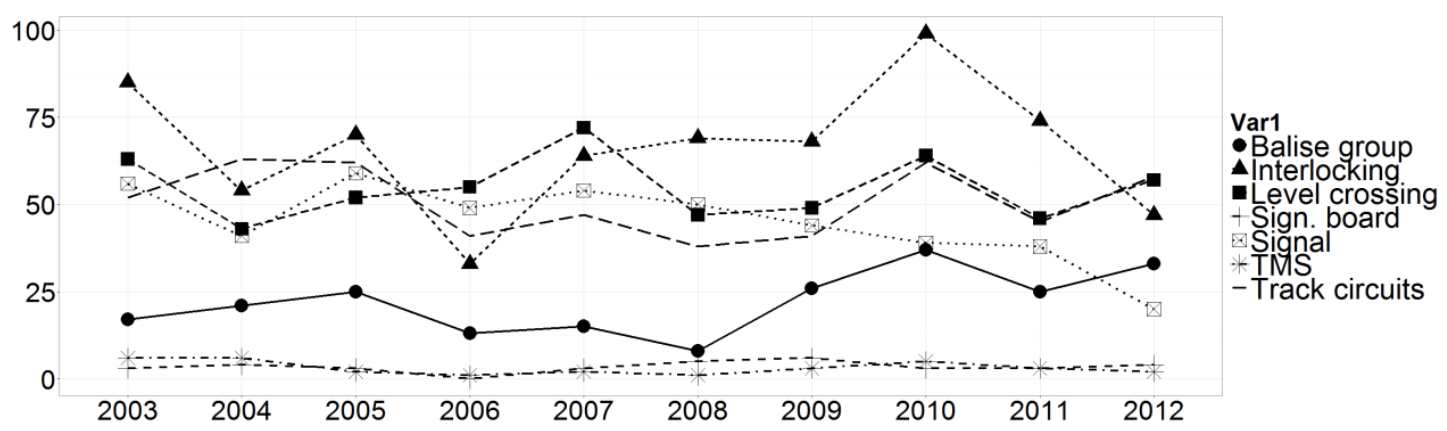

Figure 6: Failures of the signalling systems according to the year and system asset

When studying the failures by the year, we detected maximum value of WOs related to signalling systems during 2010, followed by an improvement. The number of WOs for interlockings were reduced significantly (see Figure 6). At the same time, we observed an increase in the number of WOs for the track circuits, level crossings and balise groups during the last years. The results shown in Figure 4a and 5 led us to focus our improvement measures on the RAMS of the following system assets: interlockings, 
level crossings and track circuits; this is because they produce around $80 \%$ of the WOs related to corrective maintenance. An improvement in the performance of these systems will improve the performance of the entire signalling system and, hence, the overall performance of the railway network.

\subsection{The parameter of failure mode}

The failure modes recorded are shown in Figure 5b. The most common values recorded for this parameter are "not defined" (26\%), "non-operative" (24\%) and "NFF" (21\%). In other words, either there was no failure or it was not possible to identify a failure (hence, if there actually was a failure, it would remain unremedied and the asset in question would fail again). Lacking the proper data can cause an increased amount of time to be devoted to corrective maintenance actions, as incorrect failure identification decreases the system availability.

Table 1. Failure modes according to the system asset affected

\begin{tabular}{|l|r|r|r|r|r|r|r|}
\cline { 2 - 8 } \multicolumn{1}{c|}{} & \multicolumn{7}{c|}{ System asset affected } \\
\hline Failure mode & Balise groups & IXLs & Level crossings & Sign. boards & Signals & TMS & Track circuits \\
\hline Electrical & 15 & 71 & 54 & 0 & 207 & 5 & 140 \\
\hline External & 1 & 4 & 9 & 1 & 4 & 0 & 1 \\
\hline Mechanical & 16 & 31 & 92 & 10 & 26 & 0 & 10 \\
\hline NFF & 80 & 86 & 107 & 2 & 66 & 6 & 162 \\
\hline Non-operative & 55 & 290 & 84 & 2 & 51 & 4 & 118 \\
\hline Not defined & 53 & 181 & 202 & 19 & 96 & 16 & 78 \\
\hline
\end{tabular}

Table 1 shows the relations between the system asset parameters that are affected and the failure modes. Based on these data, we can identify how the system assets fail. The most common value recorded for the failure mode of the interlockings is "non-operative". We can assume that one of the reasons for these results is the complexity of the system where the failure occurs in the case of the signals and the level crossings. For the balise groups and track circuits, "NFF" is the most recorded value for the failure mode.

\subsection{The parameter of cause of failure}

The types of failure causes related to the signalling systems are shown in Figure 4c. The most recorded values for this parameter are "not defined" (29\% of the WOs), "mechanical" $(23 \%)$ and "electrical" $(14 \%)$. Other values recorded for the cause of failure, such as "external", "environment" and "NFF", are also relevant, as each represents $10 \%$ of the WOs recorded. Clearly, the causes of failure of the signalling systems are quite widely distributed, and the data should be studied in more depth to find possible trends.

Different assets have different failure causes, since their architecture and operating conditions differ. Studying the causes according to the asset where the failure occurred, we can identify some trends. The different causes of failure recorded for the various system assets are shown in Table 2.

The most commonly recorded value for the cause of failure for four of the seven systems is "not defined". Signalling boards have an "electrical" cause and signals have a "mechanical" cause as the most common failure cause. Electrical causes derive from thunderstorms affecting the cable that connects the signalling board to the ground. For the signals, the mechanical causes of failure are higher because failures in assets such as the signal lamp or bulb are recorded as mechanical. 
Table 2. Cause of failure according to the system asset affected

\begin{tabular}{|l|r|r|r|r|r|r|r|}
\cline { 2 - 8 } \multicolumn{1}{c|}{} & \multicolumn{7}{c|}{ System asset affected } \\
\hline Cause of failure & Balise groups & IXLs Level crossings & Sign. boards & Signals & TMS & Track circuits \\
\hline Design & 8 & 16 & 7 & 0 & 6 & 4 & 5 \\
\hline Electrical & 14 & 166 & 43 & 14 & 53 & 6 & 47 \\
\hline Environment & 5 & 82 & 129 & 2 & 10 & 1 & 24 \\
\hline External & 16 & 22 & 99 & 0 & 17 & 0 & 105 \\
\hline Maintenance & 1 & 6 & 11 & 1 & 4 & 0 & 0 \\
\hline Mechanical & 29 & 143 & 96 & 7 & 210 & 6 & 59 \\
\hline NFF & 40 & 35 & 47 & 0 & 37 & 3 & 87 \\
\hline Not defined & 107 & 193 & 116 & 10 & 113 & 11 & 182 \\
\hline
\end{tabular}

\subsection{The parameter of corrective action}

The parameter of corrective action shows which actions were performed to restart the system when it failed. The most common actions recorded for the signalling systems (see Figure 4d) are "replacement" (31\%), "control" (24\%), "repair" (14\%) and "cleaning or removal of obstacles" (11\%). The architecture of most signalling systems is modular, allowing the replacement of an asset that fails with a new one, reducing the time to restoration.

Table 3 shows the relationship between the system assets and the corrective actions. While for the track circuits and balise groups the most common action is a control (45\% and 31\%, respectively), for the interlockings and signals, for example, replacement of the failed component is more important $(40 \%$ and $50 \%$, respectively). For the TMS, the most common corrective actions are replacement and restart, both corresponding to $29 \%$ of the WOs. Balise groups and track circuits are easily affected by environmental or external factors (they are located along the track), and their failure may not be permanent. Interlockings are designed as LRUs (lineside replacement units) to optimise maintenance while promoting replacement over repair on site to minimise the downtime of the system. Balise groups have replacement as the second most common corrective action. Since the failure of a balise can affect the operation of the track section where it is located, it is more effective to replace the balise with a new one, taking the failed one to the workshop to study the failure, thus minimising the downtime.

Table 3. Corrective action according to the system asset affected

\begin{tabular}{|l|r|r|r|r|r|r|r|}
\cline { 2 - 9 } \multicolumn{1}{c|}{ Corrective action } & \multicolumn{9}{c|}{ System asset affected } \\
\hline & $\begin{array}{c}\text { Balise } \\
\text { groups }\end{array}$ & IXLs & $\begin{array}{c}\text { Level } \\
\text { crossings }\end{array}$ & $\begin{array}{c}\text { Sign. } \\
\text { boards }\end{array}$ & Signals & TMS & $\begin{array}{c}\text { Track } \\
\text { circuits }\end{array}$ \\
\hline Adjustment/lubrication & 10 & 23 & 32 & 6 & 27 & 1 & 5 \\
\hline Cleaning /removal of obstacles & 5 & 20 & 139 & 1 & 19 & 0 & 76 \\
\hline Control & 99 & 119 & 128 & 2 & 90 & 6 & 158 \\
\hline No action & 7 & 15 & 5 & 0 & 17 & 0 & 71 \\
\hline Not defined & 2 & 8 & 5 & 1 & 7 & 0 & 5 \\
\hline Provisional repair & 0 & 26 & 9 & 2 & 3 & 4 & 19 \\
\hline Repair & 23 & 82 & 71 & 15 & 49 & 2 & 101 \\
\hline Replacement & 65 & 268 & 131 & 3 & 227 & 9 & 64 \\
\hline Restart & 9 & 101 & 28 & 4 & 11 & 9 & 10 \\
\hline SW update & 0 & 1 & 0 & 0 & 0 & 0 & 0 \\
\hline
\end{tabular}




\subsection{Relationship between the parameters "corrective action" and "failure mode"}

Table 4 shows the relationship between the parameters "corrective action" and "failure mode". The number of "NFFs" linked to the corrective action "control" contributes to making this measure one of the most commonly recorded corrective actions for the signalling systems. The most common corrective actions are a "control" and" replacement" of the asset. For example, "control" is related to the following values for "failure mode": "external", "NFF" and "not defined"; while "replacement" is associated with the following values for "failure mode": "electrical", "mechanical" and "non-operative". This is due to the modular architecture of signalling systems and the high incidence of "NFFs" and "not defined" failure modes.

Table 4. Corrective action according to the failure mode

\begin{tabular}{|lr|r|r|r|r|r|}
\cline { 2 - 7 } \multicolumn{1}{c|}{} & \multicolumn{9}{c|}{ Failure mode } \\
\hline Corrective action & Electr. & External & Mech. & NFF & Non-operative & Not defined \\
\hline Adjustment/lubrication & 21 & 0 & 11 & 2 & 12 & 58 \\
\hline Cleaning /removal of obstacles & 90 & 1 & 6 & 16 & 20 & 127 \\
\hline Control & 16 & 7 & 1 & 402 & 7 & 169 \\
\hline No action & 7 & 0 & 0 & 80 & 1 & 27 \\
\hline Not defined & 5 & 1 & 3 & 5 & 2 & 12 \\
\hline Provisional repair & 13 & 0 & 9 & 0 & 27 & 14 \\
\hline Repair & 54 & 4 & 48 & 1 & 171 & 65 \\
\hline Replacement & 267 & 2 & 91 & 1 & 325 & 81 \\
\hline Restart & 19 & 5 & 16 & 2 & 39 & 91 \\
\hline SW update & 0 & 0 & 0 & 0 & 0 & 1 \\
\hline
\end{tabular}

$10 \%$ of the WOs are related to the corrective action of "cleaning / removal of obstacles"; of these, most are either "not defined" or "electrical" failure modes. This could be due to the effect of rain and snow on operation. $53 \%$ of the WOs related to the corrective action "restart" have a failure mode of "not defined". This could be associated with the difficulty of identifying the failure mode of electronic assets. There are fewer appearances of the corrective action "provisional repair" associated with the failure modes "non operative", "not defined" and "electrical". This low rate reflects the modularity of signalling systems, for which the most common repair action is the replacement of the LRU.

\subsection{Relationship between the parameters "failure mode" and "cause of failure"}

When analysing the failure data, it is important to remember that different values can refer to the same kind of failure, since there is not any exact way to evaluate them. This can be seen when comparing the parameters "failure mode" and "cause of failure" (Table 5). For example, 37\% of the WOs where the cause of failure was recorded as "not defined" have "NFF" as the failure mode. With regard to the WOs where the failure mode was recorded as "non-operative", $32 \%$ of the failures were mechanical failures, $29 \%$ electrical failures and $16 \%$ were not defined.

$78 \%$ of the WOs related to environmental issues and $42 \%$ of the WOs related to external reasons have a failure mode of "not defined" or "non operative", possibly because it was not considered relevant to describe how the cause affected the asset when recording the WO; it was considered enough to simply state that the system asset was not working properly. 
Table 5. Failure mode according to the cause of failure

\begin{tabular}{|l|r|r|r|r|r|r|}
\cline { 2 - 7 } \multicolumn{1}{c|}{} & \multicolumn{7}{c|}{ Failure mode } \\
\hline \multicolumn{1}{c|}{ Cause of failure } & Electrical & External & Mechanical & NFF & Non-operative & Not defined \\
\hline Design & 5 & 2 & 3 & 2 & 11 & 23 \\
\hline Electrical & 62 & 1 & 15 & 3 & 172 & 90 \\
\hline Environment & 32 & 0 & 7 & 15 & 65 & 134 \\
\hline External & 73 & 5 & 66 & 7 & 62 & 46 \\
\hline Maintenance & 2 & 9 & 1 & 3 & 0 & 8 \\
\hline Mechanical & 234 & 0 & 67 & 0 & 193 & 56 \\
\hline NFF & 5 & 0 & 0 & 211 & 1 & 32 \\
\hline Not defined & 79 & 3 & 26 & 268 & 100 & 256 \\
\hline
\end{tabular}

\subsection{Relationship between the parameters of system asset affected, restoration time and} maintenance time

Table 6 shows the main parameters for the TTM, TTR and RRT for the whole signalling system; note that approximately half of the total time is due to the restoration time (46\%). A number of factors can influence these values, including failure mode identification and specification of the repair requirements, the distance to the failure location, human and/or material resources, etc. We can compare the maintainability between the different system assets by comparing the respective values of the mean time to restoration (MTTR), mean time to maintain (MTTM), mean waiting time (MWT) and mean relative time to restore (MRTTR) obtained from the empirical data.

Table 6. TTM, TTR and RRT

\begin{tabular}{|l|c|c|c|c|c|c|}
\hline & Min. & 1st Qu. & Median & Mean & 3rd Qu. & Max. \\
\hline Maintenance time (TTM, sec) & 180 & 4560 & 8700 & 16580 & 17400 & 86340 \\
\hline Restoration time (TTR, sec) & 60 & 1260 & 3060 & 6094 & 6960 & 83880 \\
\hline Relative restoration time (RRT, \%) & 0 & 19 & 43 & 45 & 70 & 100 \\
\hline
\end{tabular}

Figure 7 shows the relationship between the time variables (MTTM, MTTR and MWT) for the different system assets. As can be observed, the values for the MTTM and MWT have the same relationship in all the systems with the exception of the TMS, where the MTTR is proportionally much higher. The other outstanding result is the lower value of the MWT for the level crossings and the TMS. This is due to the criticality of these assets, as well as the ease of access of the asset locations.

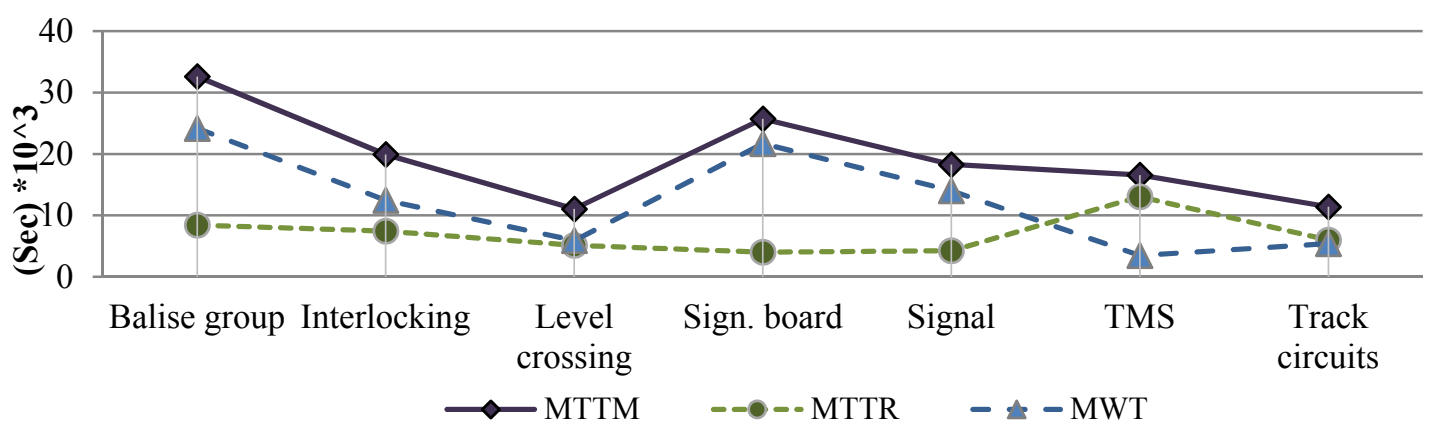

Figure 7: MTTM, MTTR and MRTTR according to the system asset 
Figure 8 visually summarises the RRT according to the system asset affected by a failure. This figure shows the maximum and minimum times spent on restore, along with the median and first and third quartiles. The density distribution is shown by the perimeter of the boxplots, and the thickness depends on the number of WOs associated with a failure of the system asset. It can be observed that the different relationships between the TTR and TTM depend on the asset affected.

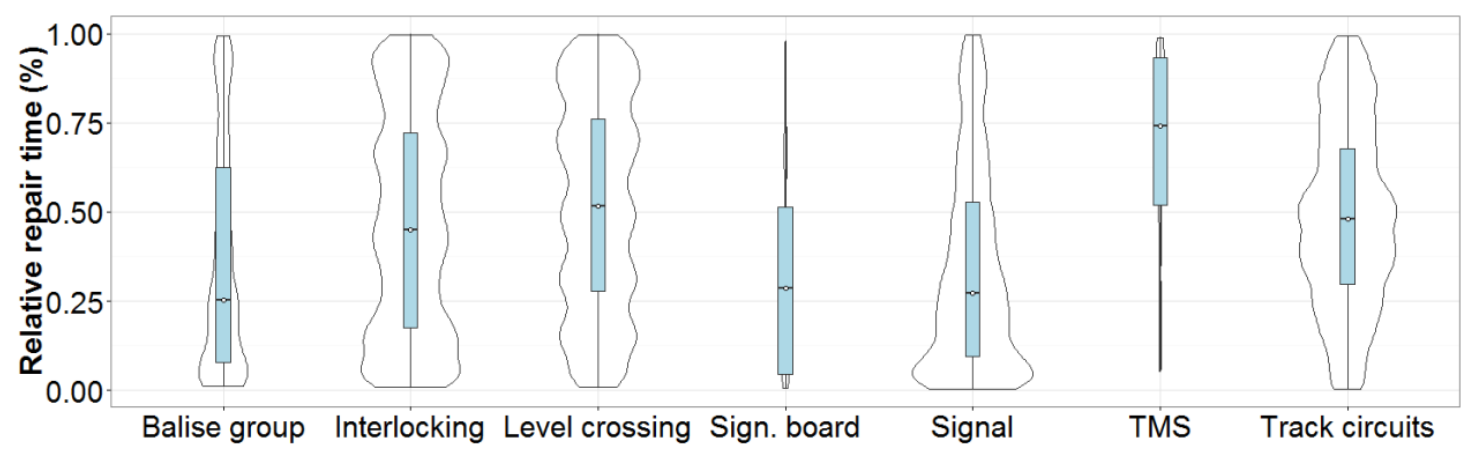

Figure 8: Relative restoration time according to the system asset

For example, the mean values for the level crossings, signals and signalling boards are quite similar and slightly lower than those for the other assets. It is possible that failures of these systems are easier to identify, since their architecture is simpler than that of systems such as interlockings or the TMS. Systems with a more mature design (i.e. a design which has not been modified to a great extent) are more familiar to the maintenance personnel than other systems; hence, the personnel's knowledge of the failure modes and their corresponding corrective actions is greater. For other systems, such as the TMS, the time to restore is higher, mainly due to the complexity of the architecture, which hinders failure identification and restoration. Some systems show an increase in the number of WOs for low restore times (e.g. the level crossings, signals and track circuits). One possible cause is the ease of identification of failures and the quickness of restorations of these systems (e.g. replacing the lamp of a signal); another possible cause is the impossibility of finding failures (NFF) on occasions.

For the system assets mostly affected by mechanical failures (e.g. signals and signalling boards), the relative restoration time is proportionally smaller and the distribution of the relative restoration time decreases when the values of TTR and TTM are more similar. Mechanical failures may be easier to identify; assets prone to mechanical failure also have a simpler architecture which facilitates repair or replacement, reducing the TTR. The balise groups also have a smaller relative restoration time, even though most of the failures are electronically based, due to the simplicity of their architecture.

For the electronically based system assets with a more complex architecture (e.g. interlockings and the traffic management system), the relative restoration time is proportionally higher than that for the mechanically based assets, and the distribution of the relative restoration time does not show a trend. Arguably, more time is spent on identifying the failure that has occurred and finding the proper corrective action.

"NFFs" are more common for electronically based systems and the architecture of these systems is more complex. Better knowledge of the systems to be maintained can reduce the time needed to identify the required corrective maintenance action in these cases. 


\subsection{Relationship between the parameters of symptom and system asset affected}

We studied the relationship between the parameters "symptom" and "system asset affected" to determine how much information could be extracted from the former. When a WO is opened, there is a symptom indicating where the failure has occurred. Failures are usually identified by the train driver, and it is not always possible for them to make an accurate identification of the failure, since failures of different systems may have the same failure effect (e.g. it can be difficult for a train driver to differentiate between a failure in the track circuit and a signal failure). The values of the "symptom" parameter are associated with the different systems; therefore, they may differ from what was reported when the WO was opened and from the system where the failure actually occurred.

To maximise the usability of the symptom parameter for giving information on the real failure, we grouped the systems into more generic groups, such as signalling systems (including track circuits, signals, interlockings, etc.), power and electric systems (e.g. transformers, substations, etc.), telecommunication systems (e.g. radio, telephony, signal cable, etc.) and track systems (turnouts, rail, etc.). When the system was not defined, it was classified as "other systems" and, when no fault was found, it was classified as a system with "NFF". Figure 9 shows the relationship between the symptoms and the system groups affected; the identification given by the symptom mostly relates to the system asset affected.

Having a more general classification of the symptoms may result in better accuracy; in addition, data classified in this manner can be used by the maintenance personnel. Therefore, using broader groups for the symptom classification can give the maintenance personnel a better initial idea of which technology has failed.

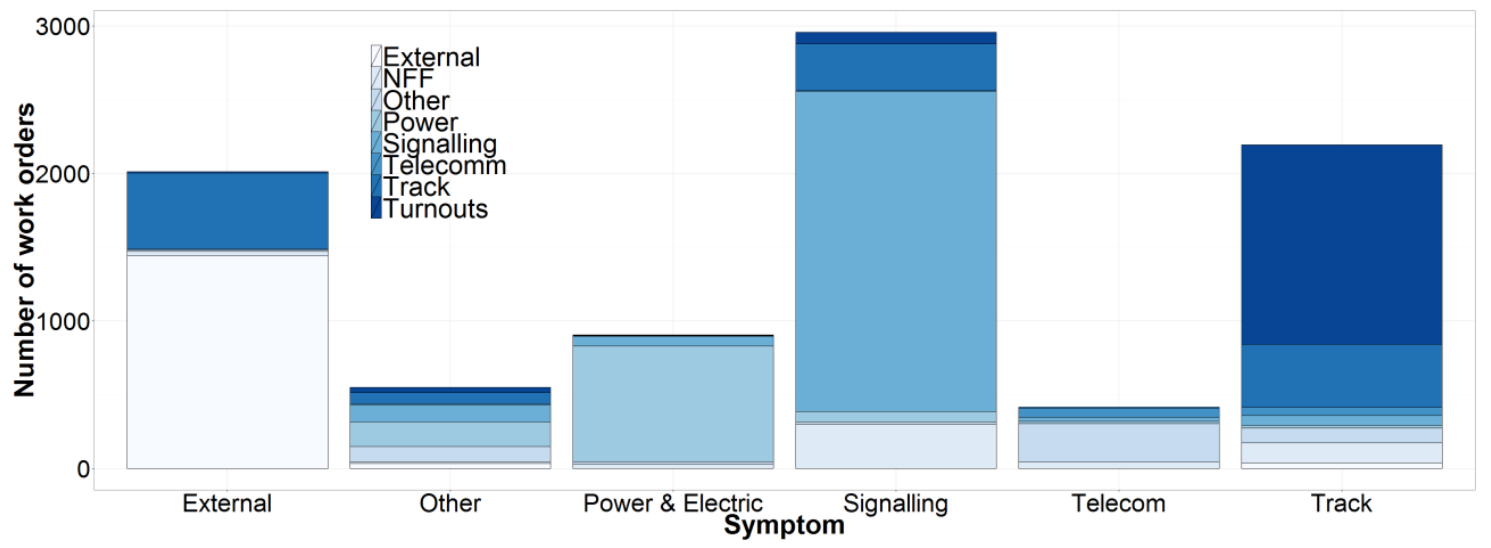

Figure 9: The system where the failure actually occurred related to the symptom

\section{DISCUSSION}

Based on the number of WOs related to failures, we conclude that signalling systems play an important role in the dependability of the railway system. Interlockings, level crossings and track circuits are the systems most affected by failures and cause most of the WO actions. Improving the performance of these systems will improve the overall performance of the railway network.

"Non operative", "not defined" and "NFF" are the most common values recorded for the failure mode for signalling systems. There are two possible reasons for this: either there was no failure or it was not possible to identify a failure (hence, if there actually was a failure, it would remain unremedied and the 
asset in question would fail again). The most common value recorded for the cause of failure is "not defined". The comparison of the number of WOs for each system and the real failure recorded shows that the more complex the system is, the more often a clear failure mode is not identified. Identifying failures in electronically based systems presents some difficulty, since aging in such systems is not directly visible (unlike mechanical fatigue). This can be seen in the high number of WOs with the following values recorded for the failure mode: "NFF", "not defined" or "non-operative"; these WOs concern failures for which the failure mode was not identified, and either no action was taken or the component was replaced. These WOs require extra time for corrective maintenance due to the time spent trying to identify a failure (sometimes unsuccessfully).

The complexity of the system and the maturity of the design architecture (e.g. it is easier to identify failures in a well-known system) play an important role when identifying failure and performing corrective maintenance actions. Better knowledge of the systems to be maintained can reduce the time needed to identify the required corrective maintenance action.

Factors such as environmental conditions or electromagnetic compatibility (EMC) can affect the normal operation of the assets, producing random failures, for which it is difficult to identify the failure cause and mode. Better failure identification would lead to better preventive measures, reducing the occurrence of failures or minimising their effects.

Studying the relationship between the corrective actions and the systems affected allows us to examine the maintainability of the various systems, and to propose improvements to reduce the time for corrective maintenance actions and increase the efficiency and efficacy of such actions. A review of the corrective maintenance procedures can help to achieve this goal.

The study of failure modes and their occurrence allows a better understanding of the reliability of the system, allowing the possibility of developing hybrid reliability models combining physics of failure and data driven models to study failure prognosis and remaining useful life. This model, together with other methodologies such as FMECA and FTA, allows companies to review their preventive maintenance inspections, with special emphasis on the most frequent failure modes. Finding the link between failure modes and failure causes assists in reviews of preventive maintenance and CBM, yielding insight into how operational and external factors affect the reliability of the system and suggesting ways to either minimise their effect or monitor them to adjust the estimated remaining useful life. Studying the causes of failure can facilitate a better understanding of the failure modes, even on WOs where little information is recorded (e.g. in the case of "not defined" and "non operative"). Linking the failure modes to the failure causes and corrective actions performed can help to reduce the time for failure identification, which leads to a reduction in the time needed to perform the corrective maintenance action (leading to improved availability).

From our observations of the time to maintain, the time to restoration and the relative restoration time, we conclude that, depending on the system asset, a number of different factors can influence the times required to maintain and to restore. Analysing the values for the factors affecting maintenance performance for each system can help reduce the times spent on corrective maintenance, thus improving the dependability of the system. Proposals for improvement depend on the focus; e.g. one can reduce the waiting time by improving the maintenance support, and one can decrease the restoration time by striving for a more efficient corrective maintenance performance, etc.

Depending on the system asset, different factors can influence the time to maintain and to restore for different reasons; examples of such factors are failure mode identification, specification of the repair requirements, the distance to the failure location, human and/or material resources, etc. Analysing the 
values of the factors that affect the maintenance performance for each system can help to reduce the time spent on corrective maintenance and improve the dependability of the system.

\section{CONCLUSIONS}

The purpose of this paper was to propose a data-driven decision support model which would integrate the various parameters of corrective maintenance data and study maintenance performance by considering different RAMS parameters. To develop our model we considered the different parameters recorded on the corrective maintenance WOs as the inputs for our model. The output of the model is the relations between the different parameters and a presentation of the relations which occur more frequently. This makes it possible to review the current maintenance policies and propose continuous improvements depending on the current performance. A limitation of the model is its dependence on the quality of the data recorded on the WOs. Depending on the quality of the input data, the reliability of the output for the decision support process can vary.

The proposed model is based on failure analysis of historical events in the form of corrective maintenance actions. It has been validated with corrective maintenance data from a specific case study. We have focused on signalling systems for two reasons. Firstly, the failure of a signalling asset may mean that the railway section where it is located will be not fully operative until the failure has been repaired (since safety cannot otherwise be ensured); hence, the availability of the whole railway section will be affected. Secondly, signalling systems receive a great deal of corrective maintenance WOs ( $27 \%$ in our case study).

Implementation of the proposed decision support model has shown that it can be successfully applied, with the following results:

- The model treats the failure occurrence from a holistic perspective; it integrates the information recorded in the different parameters of the corrective maintenance WOs.

- The model is based on empirical data and can therefore be used to validate results from other methodologies.

- The model allows companies to review how changes in maintenance policies (e.g. scheduled maintenance and inspection procedures) affect corrective maintenance performance.

- New policies can be oriented to a reduction of the most common causes of failure and to an optimisation of the most frequent corrective actions to reduce the time spent on maintenance.

- The need to improve failure identification and reduce the number of WOs with "not defined", "non-operative" and "NFF" recorded for the failure mode is indicated by the number of WOs with these values recorded. Improvements in knowledge transfer and information logistics could reduce them.

- The model links failure modes and causes of failure, establishing the basis for possible future improvements, such as the implementation of condition-based maintenance (e.g. condition-based maintenance of track circuits depending on the rainfall).

- The model identifies the assets that affect railway availability most; improving their reliability will maximise the global benefits.

Signalling systems are designed based on the "fail safe" mode; a failure can mean that the railway section where they are located will be not fully operative until the failure has been repaired. Hence, the failure of a single component can affect the availability of the whole railway network. The model identifies which systems are more likely to fail, the causes of failure and the most common corrective actions. Maintenance policies can be proposed to improve the reliability and availability of these systems.

From the results of the case study, we conclude that signalling systems play an important role in the dependability of the railway system, and this is particularly true of such assets as interlockings, track 
circuits and level crossings. We also find that "non-operative", "not defined" and "NFF" are the most common values recorded for the failure mode. Further research on the NFF phenomena can help to optimise maintenance performance and reduce the corrective maintenance WOs.

For the analysis performed in the research study presented herein, we assumed that all the failures were recorded in the corrective maintenance database. Since this research is based on empirical data, the results are limited to the information that could be obtained from the recorded data. Further research can reduce these limitations and examine the results more closely.

\section{FUNDING}

This research received financial support from Luleå Railway Research Center (JVTC) and Trafikverket.

\section{ACKNOWLEDGEMENTS}

The authors gratefully acknowledge the support of Trafikverket and Luleå Railway Research Center during this research.

\section{REFERENCES}

[1] Zoeteman A. Life cycle cost analysis for managing rail infrastructure, concept of a decision support system for railway design and maintenance. European Journal of Transport and Infrastructure Research 2001, 1(4): 391-413.

[2] Wilson A (ed). Asset maintenance management. Alden Press, Oxford, UK, 1999.

[3] EN 50126 (1999), Railway Applications - The specification and demonstration of Reliability, Availability, Maintainability and Safety (RAMS), European Committee for Electrotechnical Standardization (CENELEC), Brussels, Belgium.

[4] Patra AP. Maintenance decision support models for railway infrastructure using RAMS \& LCC analyses. $\mathrm{PhD}$ thesis, Luleå University of Technology, Luleå, Sweden, 2009.

[5] Granström R and Söderholm P. Condition monitoring of railway wheels and no fault found problems. International Journal of COMADEM, 2009, 12(2): 46-53.

[6] Williams R, Banner J, Knowles I, Dube M, Natishan M and Pecht M. An investigation of 'cannot duplicate' failures. Qual Reliab Eng Int 1998; 14(5):331-337.

[7] Pecht MG. Establishing a relationship between warranty and reliability. IEEE Transactions on Electronics Packaging Manufacturing 2006;29(3):184-190.

[8] Söderholm P. A system view of the No Fault Found (NFF) phenomenon. Reliab Eng Syst Saf 2007; 92(1):1-14.

[9] Hockley $\mathrm{C}$ and Phillips $\mathrm{P}$. The impact of no fault found on through-life engineering services. Journal of Quality in Maintenance Engineering 2012; 18(2):141-153.

[10] Granström R. Biannual revision of reinforced maintenance of roadside protective constructions (Halvårsutvärdering förstärkt underhåll vägskyddsanläggningar), Vectura $\mathrm{AB}$, Report, (in Swedish), 2012.

[11] Holmgren M. Maintenance-related losses at the Swedish Rail. Journal of Quality in Maintenance Engineering 2005;11(1):5-18. 
[12] De Felice F and Petrillo A. Methodological approach for performing human reliability and error analysis in railway transportation system. International Journal of Engineering and Technology 2011; 3(5):341-353.

[13] Lindgren J, Jonsson DK and Carlsson-Kanyama A. Climate adaptation of railways: lessons from Sweden. European Journal of Transport and Infrastructure Research, 2009; 9 (2): 164-181.

[14] Stenström C, Famurewa SM, Parida A and Galar D. Impact of cold climate on failures in railway infrastructure. In: MPMM 2012: The 2nd International Congress on Maintenance Performance Measurement \& Management Conference Proceedings, 12-13th Sep, 2012, University of Sunderland, UK.

[15] Kumar S, Espling U and Kumar U. Holistic procedure for rail maintenance in Sweden. Proc Inst Mech Eng Pt F: J Rail Rapid Transit 2008; 222(4):331-344.

[16] Berggren EG. Efficient track maintenance: methodology for combined analysis of condition data. Proc Inst Mech Eng Pt F: J Rail Rapid Transit 2010;224(5):353-360.

[17] Pecht M and Ramappan V. Are components still the major problem: a review of electronic system and device field failure returns. IEEE Transactions on Components, Hybrids, and Manufacturing Technology 1992;15(6):1160-1164.

[18] Galar D, Gustafson A, Tormos B and Berges L.Maintenance decision making based on different types of data fusion. Eksploatacja i Niezawodnosc 2012;14(2):135-144.

[19] Zhou Y, Li, Q and Zuo Y. Fault knowledge management in aircraft maintenance. In: Proceedings of the 8th International Conference on Reliability, Maintainability and Safety ICRMS; 2009:645-649.

[20] EN 50129 (2003), Railway Applications - Communication, signalling and processing systems. Safety related electronic systems for signalling, European Committee for Electrotechnical Standardization (CENELEC), Brussels, Belgium.

[21] Trafikverket. Manual - use of 0felia for analysts (Handledning - att använda Ofelia för analytiker). Report, Trafikverket, Borlänge, (in Swedish), 2010.

[22] Trafikverket. Failure reporting for the railway infrastructure (Felrapportering inom järnvägsinfrastruktur). Regulation BVF 808.20, Trafikverket, Borlänge, (in Swedish), 2011.

[23] IEC 61703 (2001), Mathematical Expressions for Reliability, Availability, Maintainability and Maintenance Support Terms. International Electrotechnical Commission, Geneva, Switzerland.

[24] Trafikverket. Railway infrastructure architecture within Trafikverket (Anläggningsstruktur järnväg inom Trafikverket). Standard BVS 811, Trafikverket, Borlänge, (in Swedish), 2012.

[25] Teetor P. R cookbook. 1st ed. O’Reilly Media, Sebastopol, Canada, 2011.

[26] IEC 60300, (2004), IEC 60300 (3-14): Dependability Management - Part 3-14: Application Guide Maintenance and Maintenance Support, International Electrotechnical Commission, Geneva.

[27] Patra AP, Dersin P, Kumar U. Cost effective maintenance policy: A case study. International Journal of Performability Engineering 2010; 6(6):595-603

[28] Patra AP, Söderholm P, Kumar U. Uncertainty estimation in railway track life-cycle cost: A case study from Swedish National Rail Administration. Proc Inst Mech Eng Pt F: J Rail Rapid Transit $2009 ; 223(3): 285-293$. 